\title{
Global Exponential Stability of A Class of Neural Networks With Unbounded and Varying Delays
}

\author{
Dianbo Ren ${ }^{1,2}$ Lan Long ${ }^{3}$ Jiye Zhang ${ }^{1}$ \\ ${ }^{1}$ State Key Laboratory of Traction Power, Southwest Jiaotong University, Chengdu 610031, P. R. China \\ ${ }^{2}$ School of Information Science and Technology, Southwest Jiaotong University, Chengdu 610031, P. R. China \\ ${ }^{3}$ Department of Mathematics and Physics, Chongqing University of Science and Technology,
}

Chongqing 400042, P. R. China

\begin{abstract}
Based on the theory of topological degree and properties of M-matrix, by constructing proper vector Lyapunov functions, the existence and uniqueness of the equilibrium point and its global exponential stability are investigated for a class of neural networks with unbounded and varying delays. Without assuming the boundedness and differentiability of the activation functions, several new sufficient criterions for ascertaining the existence, uniqueness and global exponential stability of the equilibrium point of such neural networks are obtained. Since the criterion is independent of the delays and simplifies the calculation, it is easy to test the conditions of the criterion in practice. An example is given to demonstrate the feasibility of the criterion.
\end{abstract}

Keywords: Neural networks, Globally exponential stability, Vector Lyapunov function, Unbounded delays

\section{Introduction}

In recent years, there has been increasing interest in the potential applications of the dynamics of artificial neural networks in many areas and has been extensively studied in many literature. It is well known that the signal transmissions in biological neural systems have time delays, and delays also exist in implementation of neural networks due to finite switching speed of circuit elements. Because the existence of time delays frequently causes oscillation or instability in neural networks, the study of stability of neural networks with delays is practically required. As a result, many criteria for testing the global stability of neural networks with delays have been derived; see, for example, [1-8]. Though delays arise frequently in practical applications, it is difficult to measure them precisely. In most situations, delays are variable, and in fact unbounded. Some results on the stability of neural networks involving unbounded time delays are given in [9-16], and the problem of exponential stability for neural networks with unbounded delays is studied in [14]-[16].

To the best of our knowledge, recurrent neural networks with both unbounded and variable delays are seldom considered. However, unbounded delays in recurrent neural networks are more common in practice. Therefore, the studies of recurrent neural networks with both variable and unbounded delays are more important than those with constant or variable delays. Some conditions ensuring the globally asymptotic or exponential stability of recurrent neural networks with variable time delays are given in [6-8], but in which the unbounded delays were not involved.

In this paper, on the basis of the structure of Hopfield neural networks and recurrent neural networks, we consider a class of general neural networks involving both unbounded and variable time delays. By constructing proper nonlinear integro-differential inequalities, applying M-matrix theory and vector Lyapunov function method, we obtain sufficient conditions for existence, uniqueness of the equilibrium point and its globally exponential stability of the class of neural networks with unbounded and variable time delays.

\section{Notations and preliminaries}

For convenience, we introduce some notations. $u=\left(u_{1}, \cdots, u_{n}\right)^{\mathrm{T}} \in R^{n}$ denotes a column vector. $|u|$ denotes the absolute-value vector given by $|u|=\left(\left|u_{1}\right|, \cdots,\left|u_{n}\right|\right)^{\mathrm{T}},\|u\|$ denotes a vector norm defined by $\|u\|=\left(u_{1}^{2}+\cdots+u_{n}^{2}\right)^{1 / 2}$. For matrix $A=\left(a_{i j}\right)_{n \times n}, A^{\mathrm{T}}$ denotes the transpose of $A, A^{-1}$ denotes the inverse of $A,[A]^{s}$ is defined as $[A]^{s}=\left(A^{\mathrm{T}}+A\right) / 2$, and $|A|$ denotes absolute-value matrix given by $|A|=\left(\left|a_{i j}\right|\right)_{n \times n},\|A\|$ denotes a matrix norm defined by $\|A\|=(\max \{\lambda: \lambda$ is an eigenvalue of $\left.\left.A^{\mathrm{T}} A\right\}\right)^{1 / 2}$.

The neural networks with variable and unbounded time delays investigated in this paper can be described by the following integro-differential equations:

$$
\frac{\mathrm{d} u_{i}(t)}{\mathrm{d} t}=-d_{i}\left(u_{i}(t)\right)
$$




$$
\begin{aligned}
& +\sum_{j=1}^{n}\left[a_{i j} f_{j}\left(u_{j}(t)\right)+b_{i j} g_{j}\left(u_{j}\left(t-\tau_{i j}(t)\right)\right)\right] \\
& +\sum_{j=1}^{n} c_{i j} \int_{-\infty}^{t} k_{i j}(t-s) g_{j}\left(u_{j}(s)\right) \mathrm{d} s+J_{i},
\end{aligned}
$$

where $u_{i}$ is the state of neuron $i, i=1,2, \cdots, n$, and $n$ is the number of neurons.

$$
A=\left(a_{i j}\right)_{n \times n}, B=\left(b_{i j}\right)_{n \times n}, C=\left(c_{i j}\right)_{n \times n},
$$

are the connection matrices.

$$
\begin{gathered}
f(u)=\left(f_{1}\left(u_{1}\right), f_{2}\left(u_{2}\right), \cdots, f_{n}\left(u_{n}\right)\right)^{\mathrm{T}}, \\
g(u)=\left(g_{1}\left(u_{1}\right), g_{2}\left(u_{2}\right), \cdots, g_{n}\left(u_{n}\right)\right)^{\mathrm{T}},
\end{gathered}
$$

are the activation function of the neurons.

$$
J=\left(J_{1}, J_{2}, \cdots, J_{n}\right)^{\mathrm{T}},
$$

is the constant input vector. The delays $0 \leq \tau_{i j}(t) \leq \tau$ ( $i, j=1,2, \cdots, n)$ are bounded functions, the kernels $k_{i j}:[0, \infty) \rightarrow[0, \infty)(i, j=1,2, \cdots, n)$ are piecewise continuous on $[0, \infty)$ and satisfy

$$
\int_{0}^{\infty} \mathrm{e}^{\beta s} k_{i j}(s) \mathrm{d} s=p_{i j}(\beta), i, j=1,2, \cdots, n .
$$

where $p_{i j}(\beta)$ are continuous functions in $[0, \delta), \delta>0$, and $p_{i j}(0)=1$.

The initial conditions of Eqs. (1) are of the form $u_{i}(s)=\phi_{i}(s), s \leq 0$, where $\phi_{i}$ is bounded and continuous on $(-\infty, 0]$.

In this paper, we also make the following assumptions:

Assumption 1. $d_{i}$ is continuous on $R$, and for all $x_{i}, y_{i}, \frac{d_{i}\left(x_{i}\right)-d_{i}\left(y_{i}\right)}{x_{i}-y_{i}}>D_{i}>0, i=1,2, \cdots, n$.

Assumption 2. For each $j \in\{1,2, \cdots, n\}$, the activation functions $f_{j}: R \rightarrow R$ and $g_{j}: R \rightarrow R$ are globally Lipschitz continuous with Lipschitz constants $L_{j}>0$ and $K_{j}>0$. i.e., for all $x_{j}, y_{j}$,

$$
\begin{aligned}
& \left|f_{j}\left(x_{j}\right)-f_{j}\left(y_{j}\right)\right| \leq L_{j}\left|x_{j}-y_{j}\right|, \\
& \left|g_{j}\left(x_{j}\right)-g_{j}\left(y_{j}\right)\right| \leq K_{j}\left|x_{j}-y_{j}\right| .
\end{aligned}
$$

In the following, we let

$$
\begin{gathered}
D=\operatorname{diag}\left(D_{1}, D_{2}, \cdots, D_{n}\right), \\
L=\operatorname{diag}\left(L_{1}, L_{2}, \cdots, L_{n}\right), \\
K=\operatorname{diag}\left(K_{1}, K_{2}, \cdots, K_{n}\right) .
\end{gathered}
$$

Ref. [13] assume that $d_{i}$ is differentiable and $g_{j}$ satisfy the following condition

$$
0 \leq\left(g_{j}\left(x_{j}\right)-g_{j}\left(y_{j}\right)\right)\left(x_{j}-y_{j}\right) \leq K_{j}\left(x_{j}-y_{j}\right)^{2} .
$$

It is obviously that the assumptions on functions $d_{i}$ and $g_{j}$ in Ref. [13] are more strict than Assumption 1 and Assumption 2.

To obtain our results, we give the following definitions and lemmas.
Definition 1 The equilibrium point $u^{*}$ of (1) is said to be globally exponentially stable, if there exist constants $\lambda>0$ and $M>0$ such that

$\left|u_{i}(t)-u_{i}^{*}\right| \leq M\left\|\phi-u^{*}\right\| \mathrm{e}^{-\lambda t}$, for all $t \geq 0$, where $\left\|\phi-u^{*}\right\|=\max _{1 \leq i \leq n} \sup _{s \in(-\infty, 0]}\left|\phi_{i}(s)-u_{i}^{*}\right|$.

Definition 2[17]. A real $n \times n$ matrix $A=\left(a_{i j}\right)$ is said to be an M-matrix if $a_{i j} \leq 0, i, j=1,2, \cdots, n, i \neq j$, and all successive principal minors of $A$ are positive.

Lemma 1[18]. Let $A=\left(a_{i j}\right)$ be a matrix with nonpositive off-diagonal elements. Then the following statements are equivalent:

(i) $A$ is an M-matrix;

(ii) The real parts of all eigenvalues of $A$ are positive;

(iii) There exists a vector $\xi>0$, such that $\xi^{\mathrm{T}} A>0$;

(iv) $A$ is nonsingular and all elements of $A^{-1}$ are nonnegative;

(v) There exists a positive definite $n \times n$ diagonal matrix $Q$ such that matrix $A Q+Q A^{\mathrm{T}}$ is positive.

Lemma 2[12]. If $H(x) \in C^{0}$ satisfies the following conditions: then $H(x)$ is a homeomorphism of $R^{n}$ onto itself.

(i) $H(x)$ is injective on $R^{n}$;

(ii) $\lim _{\|x\| \rightarrow \infty}\|H(x)\| \rightarrow \infty$.

\section{Existence and uniqueness of the equilibrium point}

The purpose of the present section is to give a sufficient condition ensuring the existence and uniqueness of the equilibrium point with respect to the class of unbounded activation functions and every input vector. Using the vector field associated with (1), we define a map as follows

$$
H(u)=-d(u)+A f(u)+(B+C) g(u)+J,
$$

where

$$
d=\operatorname{diag}\left(d_{1}, d_{2}, \cdots, d_{n}\right), H=\left(H_{1}, H_{2}, \cdots, H_{n}\right)^{\mathrm{T}} .
$$

It is well known that if $H(u)$ is a homeomorphism of $R^{n}$, then system (1) has a unique equilibrium point.

Theorem 1. If Assumption 1 and Assumption 2 are satisfied, and $D-|A| L-(|B|+|C|) K$ is an Mmatrix, then system (1) has a unique equilibrium point $u^{*}$.

Proof. In order to prove that system (1) has a unique equilibrium point $u^{*}$, it is only to prove that $H(u)$ is a homeomorphism on $R^{n}$. In the following, we shall prove that map $H(u)$ is a homeomorphism in two steps.

In the first step, we prove that $H(u)$ is an injective on $R^{n}$. For purposes of contradiction, suppose 
that there exist $x, y \in R^{n}$ with $x \neq y$, such that $H(x)=H(y)$. From (3), by Assumption 1 and Assumption 2, we get

$$
\begin{aligned}
|H(x)-H(y)|= & -[d(x)-d(y)]+A[f(x)-f(y)] \\
& +(B+C)[g(x)-g(y)] \mid \\
\geq & |d(x)-d(y)|-|A \| f(x)-f(y)| \\
& -(|B|+|C|)|g(x)-g(y)| \\
\geq & D|x-y|-|A| L|x-y| \\
& -(|B|+|C|) K|x-y| \\
= & {[D-|A| L-(|B|+|C|) K]|x-y| . }
\end{aligned}
$$

Since $D-|A| L-(|B|+|C|) K$ is an M-matrix, by Lemma 1 and the supposition $H(x)=H(y)$, from(4), we have

$$
|x-y| \leq[D-|A| L-(|B|+|C|) K]^{-1}|H(x)-H(y)|=0 .
$$

So $x-y=0$, i.e., $x=y$. From the supposition $x \neq y$, this is a contradiction. Thus map $H(u)$ is a injective.

In the second step, we prove that $\lim _{\|x\| \rightarrow \infty}\|H(u)\| \rightarrow \infty$. Let $\bar{H}(u)=H(u)-H(0)$. To prove that $H(u)$ is a homeomorphism, if only suffices to show that $\bar{H}(u)$ is a homeomorphism. Since $D-|A| L-(|B|+|C|) K$ is an M-matrix, from Lemma 1, there exists a positive define diagonal matrix $T=\operatorname{diag}\left\{T_{1}, T_{2}, \cdots, T_{n}\right\}$, such that

$$
[T(D-(|A| L-(|B|+|C|) K))]^{\mathrm{s}}>0 \text {. }
$$

Then it is easy to see that

$$
[T(-D+(|A| L+(|B|+|C|) K))]^{\varsigma} \leq-\varepsilon E_{n}<0,
$$

where $\varepsilon$ is a sufficiently small positive number and $E_{n}$ is the identity matrix. From (5), according to Assumption 1 and Assumption 2, we have

$[T u]^{\mathrm{T}} \bar{H}(u)=[T u]^{\mathrm{T}}(H(u)-H(0))$

$$
\begin{aligned}
= & {[T u]^{\mathrm{T}}[-(d(u)-d(0))} \\
& +A(f(u)-f(0))+(B+C)(g(u)-f(0))] \\
\leq & {[T u]^{\mathrm{T}}[-(d(u)-d(0))+|A \| f(u)-f(0)|} \\
& +(|B|+|C|)|g(u)-f(0)|] \\
\leq & |u|^{\mathrm{T}}[T(-D+|A| L+(|B|+|C|) K)]|u| \\
= & |u|^{\mathrm{T}}[T(-D+|A| L+(|B|+|C|) K)]^{\mathrm{s}}|u| \\
\leq & -\varepsilon\|u\|^{2} .
\end{aligned}
$$

Using Schwarz inequality, from (6), it is easy to get

$$
\varepsilon\|u\|^{2} \leq\|T\|\|u\|\|\bar{H}(u)\|,
$$

so we have

$$
\|\bar{H}(u)\| \geq \frac{\varepsilon\|u\|}{\|T\|} .
$$

It is obviously that $\|\bar{H}(u)\| \rightarrow+\infty$. Therefore we get $\|H(u)\| \rightarrow+\infty$ as $\|u\| \rightarrow+\infty$.

From steps 1 and 2, according to Lemma 2, we know that for every input $u$, map $H(u)$ is a homeomorphism on $R^{n}$, so system (1) has a unique equilibrium point. The proof is completed.

\section{Global exponential stability of neural networks}

Due to vector Lyapunov method is a powerful tool to analyze the stability of large-scale system, see [19]. In the section, we apply the idea of vector Lyapunov method to analyze the stability of neural networks (1).

Theorem 2. If Assumption 1 and Assumption 2 are satisfied, and $D-|A| L-(|B|+|C|) K$ is an Mmatrix, then system (1) has a unique equilibrium point, which is globally exponentially stable.

Proof. Since $D-|A| L-(|B|+|C|) K$ is an Mmatrix, from Theorem 1 , system (1) has a unique equilibrium point $u^{*}$. Let $x(t)=u(t)-u^{*}$, system (1) can be written as

$$
\begin{aligned}
\frac{\mathrm{d} x_{i}(t)}{\mathrm{d} t}= & -\bar{d}_{i}\left(x_{i}(t)\right)+\sum_{j=1}^{n} a_{i j} F_{j}\left(x_{j}(t)\right) \\
& +\sum_{j=1}^{n} b_{i j} G_{j}\left(x_{j}\left(t-\tau_{i j}(t)\right)\right) \\
& +\sum_{j=1}^{n} c_{i j} \int_{-\infty}^{t} k_{i j}(t-s) G_{j}\left(u_{j}(s)\right) \mathrm{d} s,
\end{aligned}
$$

where $i=1,2, \cdots, n, j=1, \cdots, n$, and

$$
\begin{gathered}
\bar{d}_{i}\left(x_{i}\right)=d_{i}\left(x_{i}+u_{i}{ }^{*}\right)-d_{i}\left(u_{i}{ }^{*}\right), \\
F_{j}\left(x_{j}\right)=F_{j}\left(x_{j}+u_{j}{ }^{*}\right)-F_{j}\left(u_{j}{ }^{*}\right), \\
G_{j}\left(x_{j}\right)=g_{j}\left(x_{j}+u_{j}{ }^{*}\right)-g_{j}\left(u_{j}{ }^{*}\right) .
\end{gathered}
$$

The initial condition of (7) is $\psi(s)=\phi(s)-u^{*}$, $-\infty<s \leq 0$. So (7) have a unique equilibrium $x=0$. Since $D-|A| L-(|B|+|C|) K$ is an M-matrix, from Lemma 1 , there exist $\xi_{i}>0, i=1,2, \cdots, n$, such that $-D_{i} \xi_{i}+\sum_{j=1}^{n} \xi_{j}\left[\left|a_{i j}\right| L_{j}+\left(\left|b_{i j}\right|+\left|c_{i j}\right|\right)\right] K_{j}<0, i=1,2, \cdots, n$.

Constructing the function

$$
\begin{aligned}
S_{i}(\mu)= & \xi_{i}\left(-D_{i}+\mu\right)+\sum_{j=1}^{n} \xi_{j}\left[\left|a_{i j}\right| L_{j}\right. \\
& \left.+\left(\mathrm{e}^{\mu \tau}\left|b_{i j}\right|+\left|c_{i j}\right| p_{i j}(\mu)\right) K_{j}\right], i=1,2, \cdots, n .
\end{aligned}
$$

Obviously $S_{i}(0)<0$. From (2), we know $S_{i}(\mu)$ are continuous functions. So there exists a constant $\alpha>0$, such that

$$
\begin{gathered}
\xi_{i}\left(-D_{i}+\alpha\right)+\sum_{j=1}^{n} \xi_{j}\left[\left|a_{i j}\right| L_{j}\right. \\
\left.+\left(\mathrm{e}^{\alpha \tau}\left|b_{i j}\right|+\left|c_{i j}\right| p_{i j}(\alpha)\right) K_{j}\right]<0 .
\end{gathered}
$$

Let

$$
v_{i}(t)=\mathrm{e}^{\alpha t}\left|x_{i}(t)\right|, v=\left(v_{1}, v_{2}, \cdots, v_{n}\right)^{\mathrm{T}} .
$$

calculating the upper right derivative of $v_{i}(t)$ along the solutions of (7), by using Assumption 1 and Assumption 2, we get 


$$
\begin{aligned}
D^{+} v_{i}(t)= & \mathrm{e}^{\alpha t} \operatorname{sgn} x_{i}\left\{-\bar{d}_{i}\left(x_{i}(t)\right)\right. \\
& +\sum_{j=1}^{n}\left[a_{i j} F_{j}\left(x_{j}(t)\right)+b_{i j} G_{j}\left(x_{j}\left(t-\tau_{i j}(t)\right)\right)\right. \\
& \left.\left.+c_{i j} \int_{-\infty}^{t} k_{i j}(t-s) G_{j}\left(x_{j}(s)\right) \mathrm{d} s\right]\right\}+\alpha \mathrm{e}^{\alpha t}\left|x_{i}(t)\right| \\
\leq & \mathrm{e}^{\alpha t}\left\{\left(-D_{i}+\alpha\right)\left|x_{i}(t)\right|+\sum_{j=1}^{n}\left[L_{j}\left|a_{i j}\right|\left|x_{j}(t)\right|\right.\right. \\
& +K_{j}\left(\left|b_{i j}\right|\left|x_{j}\left(t-\tau_{i j}(t)\right)\right|\right. \\
& \left.\left.\left.+\left|c_{i j}\right| \int_{-\infty}^{t} k_{i j}(t-s)\left|x_{j}(s)\right| \mathrm{d} s\right)\right]\right\} \\
\leq & \left(-D_{i}+\alpha\right)\left|\mathrm{e}^{\alpha t} x_{i}(t)\right|+\sum_{j=1}^{n}\left[L_{j}\left|a_{i j}\right|\left|\mathrm{e}^{\alpha t} x_{j}(t)\right|\right. \\
& +K_{j}\left(\mathrm{e}^{\alpha \tau_{i j}(t)}\left|b_{i j}\right|\left|\mathrm{e}^{\alpha\left(t-\tau_{i j}(t)\right)} x_{j}\left(t-\tau_{i j}(t)\right)\right|\right. \\
& \left.+K_{j}\left|c_{i j}\right| \int_{-\infty}^{t} k_{i j}(t-s) \mathrm{e}^{\alpha(t-s)}\left|\mathrm{e}^{\alpha s} x_{j}(s)\right| \mathrm{d} s\right] \\
\leq & \left(-D_{i}+\alpha\right) v_{i}(t)+\sum_{j=1}^{n}\left[L_{j}\left|a_{i j}\right| v_{j}(t)\right. \\
& +K_{j} \mathrm{e}^{\alpha \tau}\left|b_{i j}\right| v_{j}\left(t-\tau_{i j}(t)\right) \\
& \left.+K_{j}\left|c_{i j}\right| \int_{-\infty}^{t} k_{i j}(t-s) \mathrm{e}^{\alpha(t-s)} v_{j}(s) \mathrm{d} s\right] .
\end{aligned}
$$

Let

$$
\begin{gathered}
\xi_{\max }=\max _{1 \leq i \leq n}\left\{\xi_{i}\right\}, \xi_{\min }=\min _{1 \leq i \leq n}\left\{\xi_{i}\right\}, \\
l_{0}=(1+\delta)\|\psi\| / \xi_{\min },
\end{gathered}
$$

where $\delta>0$ is a constant. Then, we get that

$$
v_{i}(s)=\mathrm{e}^{a s}\left|\psi_{i}(s)\right|<\xi_{i} l_{0},-\infty \leq s \leq 0, \quad i=1,2, \cdots, n .
$$

We claim that

$$
v_{i}(t)<\xi_{i} l_{0} \text {, for } t \in[0,+\infty), i=1,2, \cdots, n .
$$

If it is not true, then there exists some $i$ and $t_{1}$, such that

$$
v_{i}\left(t_{1}\right)=\xi_{i} l_{0}, D^{+}\left(v_{i}\left(t_{1}\right)\right) \geq 0, v_{j}(t) \leq \xi_{j} l_{0},
$$

for $-\infty<t \leq t_{1}, j=1,2, \cdots, n$. However, from (9) we get

$$
\begin{aligned}
D^{+}\left(v_{i}\left(t_{1}\right)\right) \leq & \left\{\xi_{i}\left(-D_{i}+\alpha\right)+\sum_{j=1}^{n}\left[L_{j}\left|a_{i j}\right|\right.\right. \\
& \left.\left.+K_{j}\left(\mathrm{e}^{\alpha \tau}\left|b_{i j}\right|+\left|c_{i j}\right| p_{i j}(\alpha)\right)\right] \xi_{j}\right\} l_{0}<0 .
\end{aligned}
$$

This is a contradiction, so $v_{i}(t)<\xi_{i} l_{0}$, for $t \geq 0$.

Furthermore,

$$
\begin{aligned}
\left|x_{i}(t)\right| & <\xi_{i} l_{0} \mathrm{e}^{-\alpha t} \\
& \leq(1+\delta)\|\psi\| \xi_{\max } / \xi_{\text {min }} \mathrm{e}^{-\alpha t} \\
& =M\|\psi\| \mathrm{e}^{-\alpha t}, i=1,2, \cdots, n, t \geq 0,
\end{aligned}
$$

where $M=(1+\delta) \xi_{\max } / \xi_{\text {min }}$. Thus, we obtain

$$
\left|u_{i}(t)-u_{i}^{*}\right| \leq M\left\|\phi-u^{*}\right\| \mathrm{e}^{-\alpha t} .
$$

From Definition 1, the equilibrium point of (1) is globally exponential stable. The proof is completed.

\section{An illustrative example}

Consider the two-dimensional neural network of the form

$$
\dot{u}_{1}(t)=-d_{1}\left(u_{1}(t)\right)+0.2 f_{1}\left(u_{1}(t)-0.3 f_{2}\left(u_{2}(t)\right)\right.
$$

$$
\begin{aligned}
& -0.4 g_{1}\left(u_{1}\left(t-\tau_{11}(t)\right)+0.3 g_{2}\left(u_{2}\left(t-\tau_{12}(t)\right)\right)\right. \\
& +0.15 \int_{-\infty}^{t} k_{1}(t-s) g_{1}\left(u_{1}(s)\right) \mathrm{d} s \\
& +0.3 \int_{-\infty}^{t} k_{2}(t-s) g_{2}\left(u_{2}(s)\right) \mathrm{d} s+J_{1}, \\
\dot{u}_{2}(t)= & -d_{2}\left(u_{2}(t)\right)+0.1 f_{1}\left(u_{1}(t)+0.2 f_{2}\left(u_{2}(t)\right)\right. \\
& +0.25 g_{1}\left(u_{1}\left(t-\tau_{21}(t)\right)-0.2 g_{2}\left(u_{2}\left(t-\tau_{22}(t)\right)\right)\right. \\
& +0.1 \int_{-\infty}^{t} k_{1}(t-s) g_{1}\left(u_{1}(s)\right) \mathrm{d} s \\
& +0.1 \int_{-\infty}^{t} k_{2}(t-s) g_{2}\left(u_{2}(s)\right) \mathrm{d} s+J_{2} .
\end{aligned}
$$

where

$$
\begin{gathered}
d_{1}(u)=2 u, d_{2}(u)=u ; k_{1}(t)=e^{-t}, k_{2}(t)=2 /\left(1+t^{3}\right) ; \\
f_{1}(u)=\sin ((\sqrt{2} / 2) u)+u, f_{2}(u)=\sin (u) ; \\
g_{1}(u)=g_{2}(u)=\left(e^{u}-e^{-u}\right) /\left(e^{u}+e^{-u}\right) .
\end{gathered}
$$

It is easy to verify that $d_{1}(u)$ and $d_{2}(u)$ satisfy Assumption 1 with $D_{1}=2, D_{1}=1 ; f_{1}(u), f_{2}(u)$ and $g_{1}(u), g_{2}(u)$ satisfy Assumption 2 with

$$
L_{1}=1+\sqrt{2} / 2, L_{2}=1 \text { and } K_{1}=K_{2}=1 \text {; }
$$

$k_{1}(t)$ and $k_{2}(t)$ satisfy initial condition (2). Thus we have

$$
\begin{gathered}
A=\left[\begin{array}{cc}
0.2 & -0.3 \\
0.1 & 0.2
\end{array}\right], B=\left[\begin{array}{cc}
-0.4 & 0.3 \\
0.25 & -0.2
\end{array}\right], C=\left[\begin{array}{cc}
0.15 & 0.3 \\
0.1 & 0.1
\end{array}\right], \\
D=\left[\begin{array}{ll}
2 & 0 \\
0 & 1
\end{array}\right], L=\left[\begin{array}{cc}
1.707 & 0 \\
0 & 1
\end{array}\right], K=\left[\begin{array}{ll}
1 & 0 \\
0 & 1
\end{array}\right] .
\end{gathered}
$$

So we obtain

$$
D-|A| L-(|B|+|C|) K=\left[\begin{array}{cc}
1.1086 & -0.9 \\
-0.5207 & 0.5
\end{array}\right]
$$

is a M-matrix, by Theorem 2, neural network (10) is globally exponential stable.

\section{Conclusion}

In this paper, applying the idea of vector Lyapunov function method and M-matrix theory, we have obtained sufficient conditions for the existence and uniqueness of the equilibrium point and its global exponential stability of a class of neural networks with variable and unbounded delays. An illustrative example is presented which demonstrate the usefulness of the proposed results. In addition, due to sufficient conditions obtained are independent of the delays, the criteria can be easily checked in practice.

\section{Acknowledgment}

This work is supported by the National Program for New Century Excellent Talents in University (No. NCET-04-0889), and Youth Science Foundation of Sichuan (No. 05ZQ026-015).

\section{References}


[1] J. Cao, A set of stability criteria for delayed cellular neural networks, IEEE Transactions on Circuits Systems-I, 48: 494-498, 2001.

[2] W. H. Chen, Z. H. Guan and X. Lu, Delaydependent exponential stability of neural networks with variable delays, Phys. Lett. A, 326: 355-363, 2004.

[3] J. Peng, H. Qiao and Z. Xu, A new approach to stability of neural networks with time-varying delays. Neural Networks, 15: 95-103, 2002.

[4] Y. Liu and W. Tang, Exponential stability of fuzzy cellular neural networks with constant and timevarying delays. Physics Letters A, 323: 224-233, 2004.

[5] J. Zhang, Globally exponential stability of neural networks with variable delays, IEEE Transactions on Circuits and Systems-I, 50(2): 288-291, 2003.

[6] J. Liang and J. Cao, A based-on LMI stability criterion for delayed recurrent neural networks, Chaos, Solitons and Fractals, 28:154-160, 2006.

[7] H. Lu, R. Shen and F. Chung, Absolute exponential stability of a class of recurrent neural networks with multiple and variable delays, Theoretical Computer Science, 344:103-119, 2005.

[8] J. Cao and J. Wang, Global asymptotic stability of a general class of recurrent neural networks with time-varying delays, IEEE Trans. Circuits Systems I, 50(1): 34-44, 2003.

[9] Z. Yi, P. A. Ping and K. S. Leung, Convergence analysis of cellular neural networks with unbounded delay, IEEE Transactions on Circuits and Systems -I, 48: 680-687, 2001.
[10] C. Feng and R. Plamondon, On the stability analysis of delayed neural networks systems, Neural Networks, 14:1181-1188, 2001.

[11] Y. Chen, Global stability of neural networks with distributed delays, Neural Networks, 15:867-871, 2002.

[12] J. Zhang and X. Jin, Global stability analysis in delayed Hopfield neural networks models, Neural Networks, 13(7): 745-753, 2000.

[13] W. Chen and W. Zheng, Global asymptotic stability of a class of neural networks with distributed delays, IEEE Transactions on Circuits and Systems -I, 53(3):644-652, 2006.

[14] Q. Zhang, X. Wei and J. Xu, Global exponential stability of Hopfield neural networks with continuously distributed delays, Physics Letters A, 315: 431-436, 2003.

[15] J. Zhang, Y. Sud and T. Iwas, Absolutely exponential stability of a class of neural networks with unbounded delay, Neural Networks, 17: 391397, 2004.

[16] X. Liao, K.Wong and C. Li, Global exponential stability for a class of generalized neural networks with distributed delays, Nonlinear Anal.: Real World Applicat., 5:527-547, 2004.

[17] D. D. Siljak, Large-scale dynamic systemsstability and structure, New York: Elsevier, 1978.

[18] A. N. Michel, J. A. Farrell and W. Porod, Qualitative analysis of neural networks, IEEE Trans. Circuits Syst., 36(2): 229-243, 1989.

[19] J. Zhang, Y. Yang and J. Zeng, String stability of infinite interconnected system, Applied Mathematics and Mechanics, 21(7): 791-796, 2000. 